\title{
Revealing the bovine embryo transcript profiles during early in vivo embryonic development
}

\author{
Maud Vallée, Isabelle Dufort, Stéphanie Desrosiers, Aurélie Labbe ${ }^{1,2}$, Catherine Gravel, \\ Isabelle Gilbert, Claude Robert and Marc-André Sirard \\ Département des Sciences Animales, Centre de Recherche en Biologie de la Reproduction, Université Laval, \\ Québec, Québec G1V 0A6, Canada, ${ }^{1}$ Epidemiology, Biostatistics and Occupational Health Department, University \\ McGill, Montreal, Québec, Canada and ${ }^{2}$ Psychiatry Department, University McGill, and Douglas Mental Health \\ University, Montreal, Québec H3A 1A2, Canada \\ Correspondence should be addressed to M-A Sirard; Email: marc-andre.sirard@fsaa.ulaval.ca
}

\begin{abstract}
Gene expression profiling is proving to be a powerful approach for the identification of molecular mechanisms underlying complex cellular functions such as the dynamic early embryonic development. The objective of this study was to perform a transcript abundance profiling analysis of bovine early embryonic development in vivo using a bovine developmental array. The molecular description of the first week of life at the mRNA level is particularly challenging when considering the important fluctuations in RNA content that occur between developmental stages. Accounting for the different intrinsic RNA content between developmental stages was achieved by restricting the reaction time during the global amplification steps and by using spiked controls and reference samples. Analysis based on intensity values revealed that most of the transcripts on the array were present at some point during in vivo bovine early embryonic development, while the varying number of genes detected in each developmental stage confirmed the dynamic profile of gene expression occurring during embryonic development. Pair-wise comparison of gene expression showed a marked difference between oocytes and blastocysts profiles, and principal component analysis revealed that the majority of the transcripts could be regrouped into three main clusters representing distinct RNA abundance profiles. Overall, these data provide a detailed temporal profile of the abundance of mRNAs revealing the richness of signaling processes in early mammalian development. Results presented here provide better knowledge of bovine in vivo embryonic development and contribute to the progression of our current knowledge regarding the first week of life in mammals.

Reproduction (2009) 138 95-105
\end{abstract}

\section{Introduction}

The first week of embryonic development is marked by a number of critical events. Maternal transcripts that support oocyte maturation and early embryonic development are synthesized and stored in the growing oocyte (Wassarman \& Kinloch 1992). In the mouse, meiotic maturation and fertilization are known to trigger a rapid degradation of maternal transcripts (Piko \& Clegg 1982, Bachvarova et al. 1985). The remaining maternal mRNAs sustain protein synthesis during the transcriptional silent phase until the newly formed embryonic genome is activated. In bovine embryos, the maternal to embryo transition (MET) occurs at the 8- to 16-cell stage (Memili et al. 1998). During the following stages, important morphological and functional changes occur to produce the blastocyst that is characterized by the presence of differentiated cellular structures namely the trophectoderm and the pluripotent cells of the inner cell mass. All these steps are likely to be associated with marked changes in gene expression profiles, or more precisely RNA abundance, since prior to the MET the detected RNAs originate from the maternal stocks rather than being the product of immediate transcription. Moreover, the need to perform a descriptive RNA abundance profiling across bovine embryonic development is ambitious since the limited amount of starting material requires the use of an amplification method that often introduce biases that consequently prevent reliable comparison between developmental stages. Indeed, the pre-amplification procedures, either PCR or in vitro transcription based, have been developed to keep the relative abundance of the transcripts within a sample, but they have not been optimized for keeping the relative abundance of starting material between samples that show a wide range of starting material quantity. This is particularly important when comparing samples naturally containing different amounts of RNA such as 
in the case of oocytes and/or early bovine embryos. Using an appropriate method that keeps the relative abundance of transcripts within and across developmental stages will provide better knowledge of the molecular mechanisms controlling in vivo embryonic development.

In the last years, numerous microarray profiling studies have been published regarding embryonic development, notably in the mouse (Hamatani et al. 2004a, 2004b, 2004c, Wang et al. 2004, Zeng et al. 2004, Zeng \& Schultz 2005, Yoon et al. 2006, Su et al. 2007). However, the unavailability of adequate sequence data for numerous species has kept the microarray technology beyond the reach of investigators working on economically and scientifically important large domestic species such as cattle, pigs, and sheep. Possible solutions to this problem is either the use of cross-species hybridizations (Robert et al. 2000, Dalbies-Tran \& Mermillod 2003, Adjaye et al. 2004, 2007, Vallee et al. 2008) or the fabrication of homemade cDNA arrays (Yao et al. 2004, Whitworth et al. 2005, Vallee et al. 2006, Dessie et al. 2007, Ghanem et al. 2007). The commercial arrays (Affymetrix) that have been made available for the cow greatly facilitate bovine gene expression profiling (Fair et al. 2007). However, even if large cohorts of genes are available on this bovine array, the gene list indicates a poor representation of known oocyte and early embryonic specific gene candidates. Similar findings were previously shown from a mouse embryo expressed sequence tag (EST) project (Ko et al. 2000) where a significant fraction of the genes expressed in early life were not represented in the collections of genes available on commercial arrays. Following this EST project, a mouse developmental array was developed (Carter et al. 2003) and was subsequently used for all transcript profiling analysis of oocytes, embryos, and stem cells (Hamatani et al. 2004a, 2004b, 2004c, Aiba et al. 2005, Vallee et al. 2008).

In this study, we present the transcript profiling of bovine in vivo early embryonic development on a bovine developmental array containing over one thousand transcripts derived from bovine oocyte and blastocyst subtracted libraries. Oocytes and embryos from three developmental stages (two-cell, eight-cell, and blastocyst) were used for this gene expression profiling. We chose to analyze embryos that developed in vivo, rather than in vitro, to study the molecular mechanisms involved during in vivo embryonic development and thus avoid the effect of culture conditions on gene expression. Results presented here will provide better knowledge of bovine in vivo embryonic development, and also be used for comparative analysis of early development in other mammalian species, including mouse and human. Therefore, it should contribute to the progression of our current knowledge regarding the earliest stages of mammalian life.

\section{Results}

\section{Array design and fabrication}

A detailed description of array design and fabrication of a preliminary version of this array (v.1.0) has been previously published (Sirard et al. 2005). The microarray platform used in this study (v.1.2) contains 1153 transcripts highly relevant to early embryonic development that were derived from four distinct subtracted libraries (the complete list is given in Supplementary Table 1, which can be viewed online at www. reproduction-online.org/supplemental/). The transcripts derived from the first subtracted library are preferentially expressed in germinal vesicle (GV) oocytes as the transcriptome was subtracted to a pool of somatic tissues (liver, kidney, spleen, muscle, and cumulus cells; Vallee et al. 2005). The second subtracted library consists of transcripts preferentially found in GV oocytes as the maternal transcriptome was subtracted using the RNA content of day 8 in vitro produced blastocysts. The third subtracted library is the reverse experiment (day 8 blastocysts - GV oocytes) producing a gene collection containing candidates expressed following the MET. Finally, the fourth subtracted library is from day 8 in vitro produced blastocysts subtracted using a sample made from a pool of somatic tissues, which produces a gene collection enriched in candidates related to the specific nature of the blastocyst. The number of transcripts derived from each subtracted library found on the microarray slide is presented in Table 1. Each clone was sequenced and spotted in four replicates on the array. Furthermore, multiple controls that can be used as positive or negative control spots were added in a randomized fashion on the array. They consist of GFP cDNA fragment, Alien 1, Alien 2, and Arabidopsis thaliana CAB1 gene from SpotReport TM cDNA Array Validation System (Stratagene, La Jolla, CA, USA), ubiquitous genes (actin, tubulin, and ubiquitin), and finally, spotting solution controls and empty controls. In this experiment, Alien 1 and Alien 2 spots were used as negative controls and GFP and CAB1 genes that were spiked in the samples prior to hybridization were used as exogenous positive controls for data normalization.

Table 1 Number of transcripts derived from each subtracted library.

\begin{tabular}{lcccc}
\hline Library \# & $\mathbf{1}$ & $\mathbf{2}$ & $\mathbf{3}$ & $\mathbf{4}$ \\
\hline Library $_{\text {name }^{\mathrm{a}}}$ & $\begin{array}{c}G V \\
\text { oocytes } \\
\text { Somatic } \\
\text { tissues }\end{array}$ & $\begin{array}{c}G V \\
\text { oocytes } \\
\text { Blastocysts }\end{array}$ & $\begin{array}{c}\text { Blastocysts } \\
\text { GV } \\
\text { oocytes }\end{array}$ & $\begin{array}{c}\text { Blastocysts } \\
\text { Somatic tissues }\end{array}$ \\
\hline $\begin{array}{c}\text { Number of } \\
\text { transcripts }\end{array}$ & 403 & 283 & 206 & 261 \\
\hline
\end{tabular}

${ }^{\mathrm{a}}$ For each library, transcripts derived from that library are preferentially expressed in the stage represented in italics. The subtraction procedure was performed with the stage below. 
RNA amplification

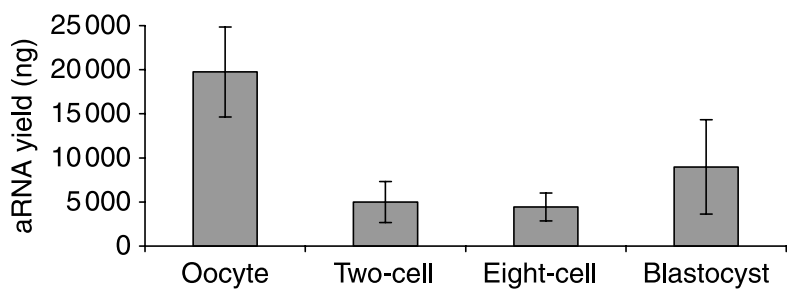

Figure 1 aRNA amplification yield. The average yield of aRNA obtained for each developmental stage after two rounds of linear amplification. Total RNA from three pools of ten oocytes or embryos was used for amplification using the RiboAmp HS RNA Amplification kit (Molecular Devices Corporation, Sunnyvale, CA, USA).

\section{Validation of linear RNA amplification}

Pools of ten oocytes or embryos were used for amplification, and, as shown in Fig. 1, the amount of amplified RNA (aRNA) generated from the restricted amplification reaction is different for each developmental stage, which is in corroboration with the natural RNA contents between these stages. During the amplification, the incubation period was limited to avoid a plateau phase, typical of any amplification method, which would hinder the capacity to perform across stage comparisons. Determination of the optimal incubation time was done by performing a time course that followed the reaction kinetics (Gilbert et al. 2009). To further validate that no distortion had occurred during the amplification reaction, quantitative PCRs were performed with four different genes from unamplified, first and second round amplification samples for all developmental stages (Fig. 2).

\section{Microarray reproducibility}

Reproducibility of microarray hybridization was verified as a quality assessment by calculating the correlation coefficients of signal intensities between replicated experiments in a pair-wise manner (Fig. 3A). The excellent mean correlation (0.98) found between replicated experiments is adding confidence in the validity of the experiment. Furthermore, an unsupervised hierarchical clustering using all genes found on the array clustered all replicates alongside their appropriate developmental stage (Fig. 3B). The clustering dendogram (Fig. 3B) reveals that the two- and eight-cell embryos are subgrouped together. By contrast, the GV oocyte and blastocyst are independently significantly different from the other developmental stages.

\section{Analysis of RNA abundance profiles in bovine in vivo embryos}

To perform the analysis of gene expression in bovine in vivo embryos, all microarray data were uploaded in the NIA array analysis tool and a threshold was established according to the plot of error function to determine which candidates were generating signals significantly above background e.g. considered as present. Genes with a mean log signal intensity above the establish threshold (log 2.5) were considered as expressed, whereas the genes with signal intensities below the threshold were designated as 'absent'. This analysis based on intensity values revealed that the majority of the genes found on our bovine developmental array are expressed at some point during in vivo
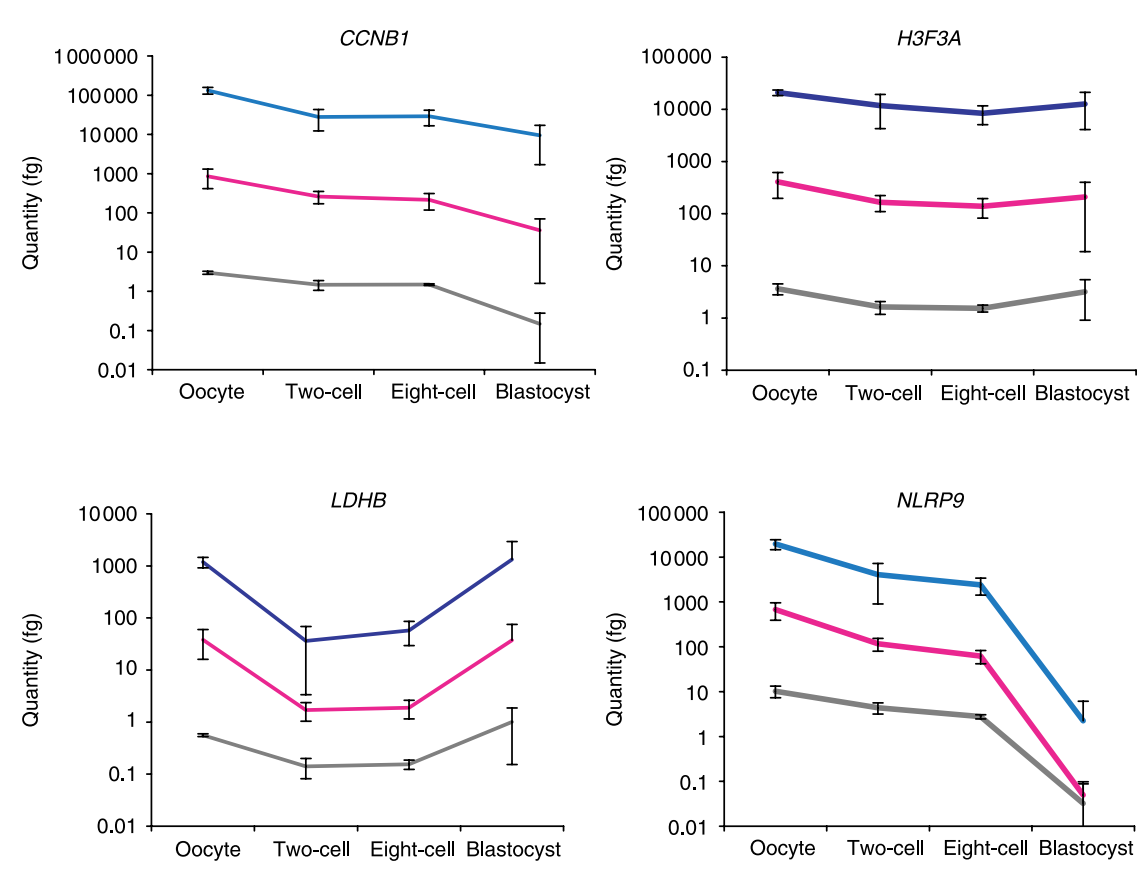

Figure 2 Efficiency assessment of the amplification procedure in preserving the relative RNA content between developmental stages. Quantification by RT-PCR of four genes in bovine oocytes and in vivo embryos. Cyclin B1 (CCNB1), $\mathrm{H} 3$ histone, family $3 \mathrm{~A}$ (H3F3A), lactate dehydrogenase $\mathrm{B}(L D H B)$, and NACHT leucine-rich repeat and PYD containing 9 (NLRP9). Reactions were performed with unamplified (gray line), first (pink line) and second round (blue line) amplification cDNA samples $(n=3)$. Shown is the relative mRNA abundance (mean \pm S.E.M). 
A

\begin{tabular}{lc}
\hline $\begin{array}{l}\text { Developmental } \\
\text { stage }\end{array}$ & $\begin{array}{l}\text { Correlation } \\
\text { coefficient }\end{array}$ \\
\hline Oocyte & 0.99 \\
Two-cell & 0.99 \\
Eight-cell & 0.98 \\
Blastocyst & 0.98 \\
\hline
\end{tabular}

B
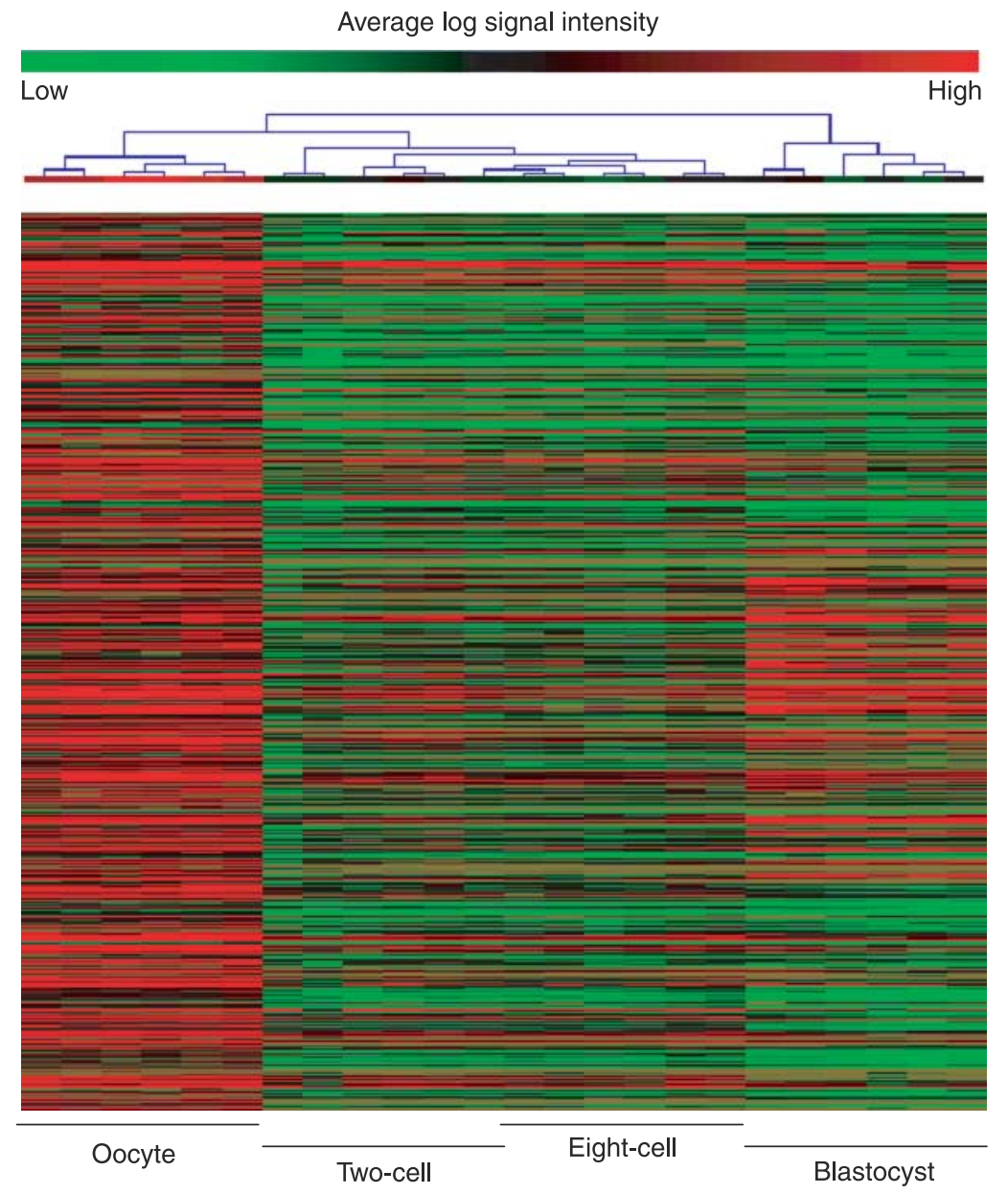

Figure 3 Microarray reproducibility. (A) Correlation coefficients of signal intensity between replicated experiments in a pair-wise manner. (B) Hierarchical clustering of replicated experiments. Colors correspond to relative RNA abundance for the detected genes, each of which is represented by one horizontal bar.

embryonic development. The number of genes detected varies between each developmental stage, confirming the dynamic profile of gene expression observed during embryonic development (Table 2).

\section{Comparative transcript abundance profiling}

To obtain a perspective on RNA abundance fluctuations, we performed a pair-wise comparison of gene expression microarray data for all developmental stages (Fig. 4). Expectedly, the pair that showed the biggest difference in the nature and abundance of transcripts was GV oocytes and blastocysts. As shown in the log ratio plot, 776 clones were overexpressed in GV oocytes and 52 were overexpressed in blastocysts (Fig. 4). Genes overexpressed in GV oocytes were predominantly derived from subtracted libraries 1 and 2 , whereas genes overexpressed in blastocysts were exclusively derived from subtracted libraries 3 and 4 . The complete list of genes overexpressed in GV oocytes compared with two-cell, eight-cell, and blastocysts can be viewed in Supplementary Table 1 . Furthermore, the comparison of two- and eight-cell embryos revealed no major difference between those two developmental stages (Fig. 4), which support the fact that they cluster together in the hierarchical clustering analysis (Fig. 3).

\section{Maternal versus embryonic transcripts}

Principal component analysis of our microarray data revealed that the majority of the genes expressed during the bovine first week of embryonic in vivo development are grouped in three main clusters corresponding to three general trends of abundance patterns (Fig. 5 and Supplementary Table 1). Additionally, classification of genes according to the Gene Ontology biological process category allowed the functional classification of genes making up the three main general trends (Khatri et al. 2002). The first two clusters present a profile 
Table 2 Genes detected in each developmental stage.

\begin{tabular}{lcc}
\hline Stage & Genes detected $^{\mathrm{a}}$ & $\mathbf{( \% )}^{\mathrm{b}}$ \\
\hline Oocyte & 1137 & 98.61 \\
Two-cell & 1038 & 90.03 \\
Eight-cell & 1055 & 91.50 \\
Blastocyst & 981 & 85.08 \\
\hline
\end{tabular}

${ }^{\mathrm{a} C l o n e s}$ with a mean log signal intensity above the calculated background threshold ( $\log 2.5$ ) were considered as expressed.

${ }^{b}$ Percentage of genes expressed on total number of transcripts on the array.

characteristic of maternal genes (Fig. 5A and B). The last cluster, corresponding to a maternal-to-embryonic expression pattern, regroups $10 \%$ of the genes in our microarray data (Fig. 5C).

\section{Validation of microarray data by quantitative RT-PCR}

Finally, confirmation of the normalized microarray data was performed by a quantitative RT-PCR analysis of four transcripts. The selection of these candidates used for validation was based on the clustering analysis described previously (Fig. 5). Two transcripts with a maternal profile, NLRP9 and CCNB1 (Fig. 5A and B), and two transcripts with a maternal-to-embryonic profile, $H 3 F 3 A$ and $L D H B$ (Fig. 5C), were chosen as representative of the major expression profiles observed. Aliquots of in vivo embryos unamplified RNA were used for the PCR amplifications and detailed information regarding the primers is presented in Table 3. Comparing the profiles generated from the PCR amplifications to the one produced by the microarray data allowed validation of our normalized microarray data. The results shown in Fig. 6 confirm the profiles of all candidates detected by microarray. Although no significant difference was observed between the developmental stages for H3F3A, the expression profile obtained from the quantitative RT-PCR was similar to the one obtained from the microarray data (Fig. 6).

\section{Discussion}

The main objective of this study was to perform a transcript abundance profiling analysis of bovine early embryonic development in vivo using a bovine developmental array. The molecular description of the first week of life at the mRNAs level is particularly challenging when considering the important fluctuations in RNA content that occur between developmental stages. Since embryos contain very little RNA for evaluation, a modified linear amplification method was used to produce aRNA in sufficient amount to perform microarray hybridizations. During the amplification, the incubation period was limited to avoid a plateau phase, typical of any amplification method, which would hinder the capacity to perform across stage comparisons. As shown, the abundance patterns of the unamplified samples throughout development are conserved after the first round of amplification and most importantly after the second round of amplification. Therefore, the modified amplification method used in this study preserved the relative RNA content between samples, which is essential to allow the production of physiologically relevant developmental stage profiles. Additionally, a key step to keep the relative initial RNA contents between samples was done during data normalization. Indeed, an equal amount of aRNA was used to prepare all microarray probes in order to avoid the bias caused by the high variability, which characterizes low intensity signals that would have been generated in a much larger proportion with the low RNA content stages. This aRNA equalization alleviates the natural RNA content differences between developmental
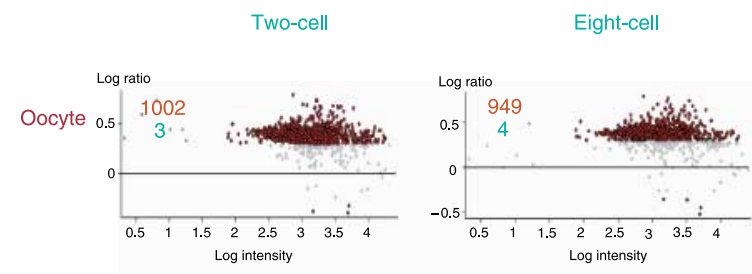

Two-cell

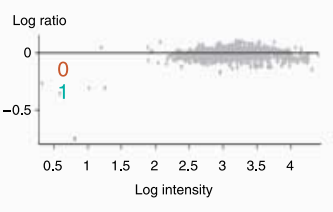

Eight-cell
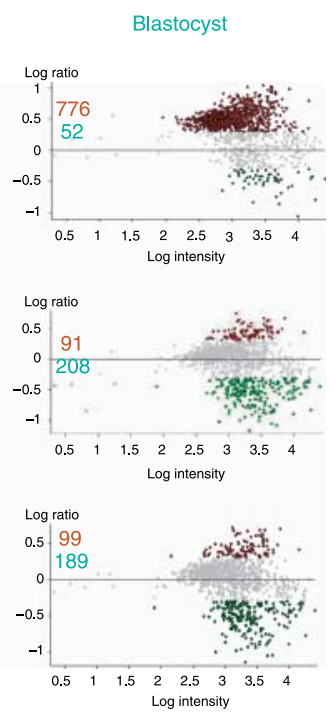

Figure 4 Mean pair-wise comparison. Scatter plots representing gene expression between each pair of developmental stages. Horizontal axis represents the average log intensity of clones, whereas a vertical axis represents the log ratio of signal intensity for each clone between one stage and another stage. Colored spots (red and green) represent clones that passed the FDR $10 \%$. Red spots represent array features with higher expression at the stage indicated on that row, whereas green spots represent array features with lower expression. The number of clones overexpressed is indicated in red and the number of clones underexpressed is indicated in green besides each scatter plot. 
A

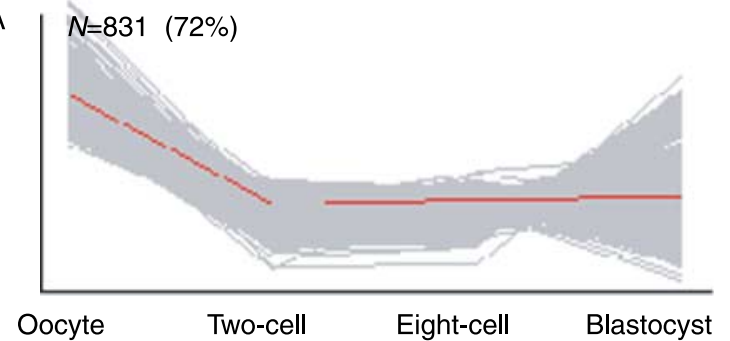

B
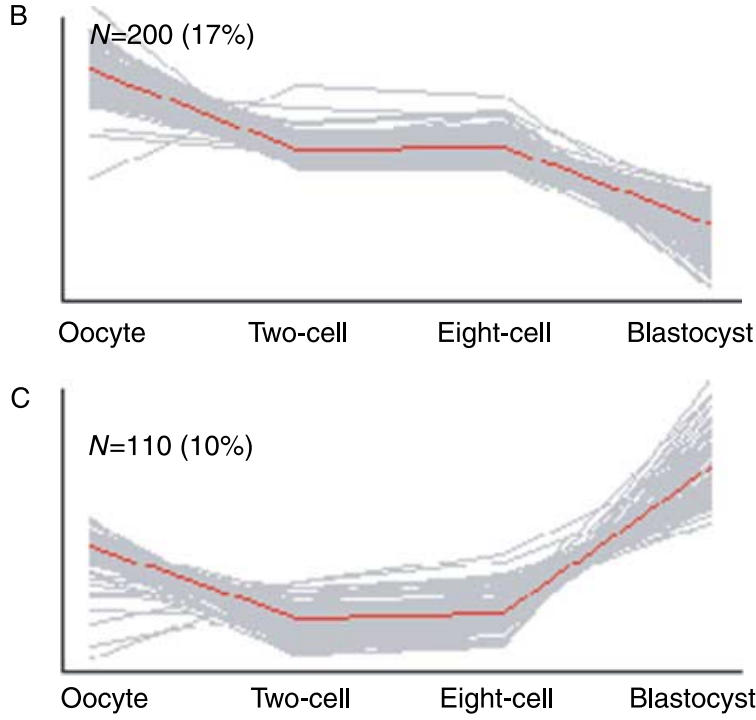

Figure 5 Clustering analysis. The NIA array analysis tool was used to perform a principal component analysis (three principal components, 1.5 -fold change, and 0.7 correlation threshold) using all genes on the array. The vast majority of present transcripts (99\%) fall within one of these clusters. Maternal profile (A and B) and maternal-to-embryonic profile $(\mathrm{C})$ are observed. Gray lines represent transcript intensities, and the red line is the average intensity of the cluster.

stages that were preserved during the pre-amplification step. In order to take this into account, following the amplification reaction, an equal amount of an in vitro produced RNA coding for GFP was spiked in every sample. Since the concentration of aRNA differed between the samples, the volume taken from each samples for hybridization proportionally differed. The signals generated by the GFP spikes were representative of the RNA yields for each developmental stage and were used to correct the data to the initial natural relative abundance between developmental stages. Failing to do so would have involved the comparison of different amount of embryos at each developmental stage, which would have compromised the capacity to perform across stage analyses. The validation of the entire data normalization procedure was performed with quality assessment of the microarray data and quantitative PCR profiles.

Unsupervised hierarchical clustering independently clustered all the replicates by their appropriate developmental stage with minimal branch tree distances. With the exception of a few genes, most of the genes behaved similarly across the three replicates for each developmental stage. The wider range of RNA abundance among replicates for some genes may reflect the asynchrony of embryo development in vivo. The clustering of the two- and eight-cell embryos from the other stages is consistent with the fact that there is very little transcription before the eight-cell stage (Memili et al. 1998). Although the depletion of maternal mRNA either through translation or RNA decay begins during oocyte maturation, destruction of these mRNAs is typically not complete until the 8- to 16-cell stages. Thus, most of the transcripts present are maternally derived. By contrast, transcripts present at the blastocyst stage are, for the most part, newly synthesized and form a distinct population compared with the one found in GV oocytes. Although the onset of embryonic transcription occurs at different stages depending on the species (Telford et al. 1990), independent studies performing hierarchical clustering of microarray experiments on mouse pre-implantation embryos also clustered developmental stages according to MET; prior to embryonic genome activation, during major reprogramming, or after embryonic genome activation (Hamatani et al. 2004a, 2004b, 2004c, Zeng et al. 2004). The concordance of the hierarchical cluster

Table 3 Primers used for validation by quantitative PCR.

\begin{tabular}{|c|c|c|c|c|c|c|}
\hline Gene name & $\begin{array}{l}\text { Gene } \\
\text { symbol }\end{array}$ & Primer sequences & $\begin{array}{l}\text { Amplicon } \\
\text { size (bp) }\end{array}$ & $\begin{array}{c}\text { Annealing } \\
\text { temperature } \\
\left({ }^{\circ} \mathrm{C}\right)\end{array}$ & $\begin{array}{c}\text { Melting } \\
\text { temperature } \\
\left({ }^{\circ} \mathrm{C}\right)\end{array}$ & $\begin{array}{l}\text { Accession } \\
\text { number }\end{array}$ \\
\hline Cyclin B1 & $C C N B 1$ & $\begin{array}{l}\text { Up: 5'-GATCAGCACTCTAGCACAGC-3' } \\
\text { Low: 5'-AAAGGATAAGTAAAAAGAACTTCAAC-3' }\end{array}$ & 181 & 52 & 81 & NM_001045872 \\
\hline $\begin{array}{l}\text { H3 histone, family } \\
\text { 3A }\end{array}$ & H3F3A & $\begin{array}{l}\text { Up: 5'-CATGGCTCGTACAAAGCAGA-3' } \\
\text { Low: 5'-AGACGCTGGAAGGGAAGTTT-3' }\end{array}$ & 213 & 55 & 86 & NM_001014389 \\
\hline $\begin{array}{l}\text { Lactate dehydro- } \\
\text { genase B }\end{array}$ & $L D H B$ & $\begin{array}{l}\text { Up: 5'-AAGTGGATTACCCAAGCACCGT-3' } \\
\text { Low: 5'-AGAAACGCCTGCCACATTCACT-3' }\end{array}$ & 181 & 59 & 84 & NM_174100 \\
\hline $\begin{array}{l}\text { NACHT, leucine-rich } \\
\text { repeat and PYD } \\
\text { containing } 9\end{array}$ & NLRP9 & $\begin{array}{l}\text { Up: 5'-TACGATGCTGGCACCAAACAGT-3' } \\
\text { Low: 5'-AGACTGACCAAGGCTTCACACA-3' }\end{array}$ & 215 & 58 & 88 & NM_001024664 \\
\hline
\end{tabular}



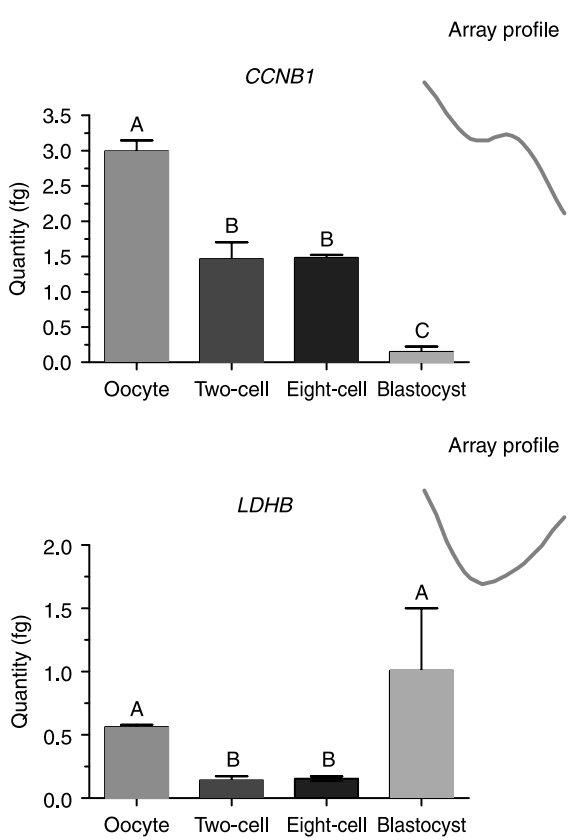
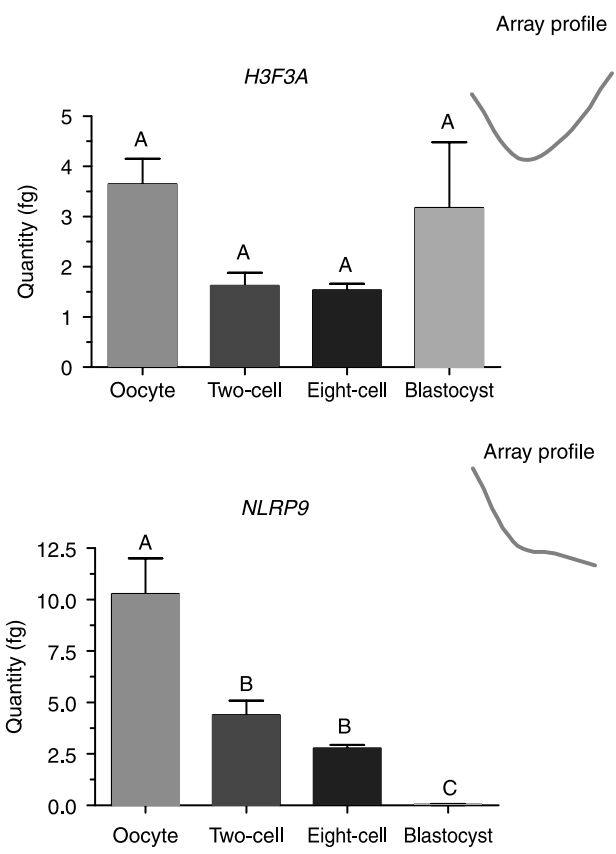

Figure 6 Microarray validation by quantitative PCR. Quantification of cyclin $\mathrm{B} 1$ (CCNB1), $\mathrm{H} 3$ histone, family $3 \mathrm{~A}(H 3 F 3 A)$, lactate dehydrogenase $\mathrm{B}$ $(L D H B)$, and NACHT leucine-rich repeat and PYD containing 9 (NLRP9) mRNA levels in bovine oocytes and embryos during in vivo embryonic development. Reactions were performed with unamplified cDNA samples $(n=3)$. Shown is the relative mRNA abundance (mean \pm s.E.M). Different letters indicate a significant difference of relative mRNA abundance $(P<0.05)$. analysis with previous results found on a different species lends confidence to the reliability of the conclusions drawn from our transcript profiling of prehatching bovine embryos.

When pair-wise comparison of genes present across all developmental stages was performed, the pair that showed the biggest difference was GV oocytes and blastocysts. Known oocyte-specific genes are included in the list of genes overexpressed in oocytes such as, ZP1, ZP2, ZP3, and FIGLA (Philpott et al. 1987, Liang et al. 1990, 1997, Epifano et al. 1995). Other genes that were previously identified in our laboratory and in others as preferentially expressed in oocytes were also present in this list such as: BTG4, HMGN2, and PAIP1 (Vigneault et al. 2004, Pennetier et al. 2005, Vallee et al. 2005). The particular expression profiles of these genes suggest a possible implication in early bovine development and embryonic genome activation. The similar profiles observed between the two- and eight-cell stages are probably due in part by the lack of genes derived from these two developmental stages found on the array but also it reflects the fact that major maternal mRNA depletion events occur before the two-cell stage, as seen in the mouse (Piko \& Clegg 1982). Following this major wave of mRNA decay, the expression profiles seem to maintain a certain stability prior to embryonic genome activation occurring at the 8to 16-cell stages (Memili et al. 1998).

Three general trends of abundance patterns among genes expressed during the bovine first week of embryonic in vivo development were uncovered by principal component analysis of our microarray data. The first two trends are characteristics of maternal genes and thus suggest an important role for these transcripts during early embryonic development. Functional classification revealed that regulation of transcription and cell cycle are the biological processes overrepresented in both of these clusters. The first cluster represents genes that are rapidly degraded following fertilization (such as NLRP9, FIGLA, ZP2, ZP3, and ZP4) and the second cluster represents progressively degrading transcripts (such as CCNB1, HMGN2, PTTG1, and BTG4). The profiles observed for NLRP9 and HMGN2 obtained using microarrays are similar to previously reported expression profiles during bovine in vitro embryonic development obtained by PCR (Vigneault et al. 2004, Dalbies-Tran et al. 2005). In the last cluster, corresponding to a maternal-to-embryonic expression pattern, some transcripts are equally abundant in oocytes and blastocysts (such as H3F3A and EEF1A1) while others are more abundant in blastocysts than in oocytes (such as $L D H B$ and HIST4A4). Functional classification revealed that translation and oxidation reduction are the biological processes represented in this last cluster. Genes found in the later cluster present a pattern characterized by a rapid decrease in maternal mRNAs after fertilization, followed by an increase in expression after embryonic genome activation, suggesting a constant role for these factors during embryonic development. Finally, a validation of the normalized microarray data by quantitative RT-PCR confirmed the profiles of four selected transcripts with either maternal or maternal-to-embryonic profiles. Moreover, this validation provided the confirmation that our microarray normalization strategy used to correct for the different amounts of RNA found in each developmental stage was successful and did not create any distortion. 
In conclusion, the study presented herein provides a profile of a unique collection of genes having their RNA present during bovine prehatching embryonic development in vivo. The strategy used in this study to correct for the varying amount of RNA found in each developmental stage, that is the use of a modified RNA amplification technique combined to a microarray normalization procedure, will provide a more accurate outlook of the transcript abundance profiling during in vivo bovine embryonic development. However, since the microarray platform used in this study covers only a portion of the genome, we are currently working on the enrichment of our bovine development microarray platform specially to include genes derived from each developmental stage. Nevertheless, results obtained in this study have proven that microarray is a powerful tool for the study of gene expression profiling during embryonic development. The major strength of this experiment relies on the fact that in vivo embryonic development was studied with a developmental array enriched with transcripts expressed during early embryonic development. This in vivo embryonic development profiling will serve as a reference for future experiments and will enable comparisons between IVF protocols, developmental stages, and even between laboratories. Finally, a better comprehension of bovine early embryonic development in vivo will contribute to the progression of our current knowledge regarding the first week of life in mammals.

\section{Materials and Methods}

\section{Oocytes and embryos collection}

Holstein and Simmental heifers were used for the oocytes and embryos collection. For the collection of GV oocytes, cumulus-oocyte complexes from 3 to $6 \mathrm{~mm}$ follicles were obtained by follicle liquid aspiration from ovaries as previously described (Vigneault et al. 2004). The oocytes were mechanically denuded by vortexing and washed several times in PBS solution to prevent cumulus cell contamination. For the collection of in vivo two- and eight-cell embryos, animals were stimulated by application of Stimufol (FSH:LH=5:1) twice a day at a dosage of $1.0 \mathrm{ml}$ for a period of four consecutive days. Following the stimulation, animals were artificially inseminated using bull spermatozoa. The embryo recovery was done by Besenfelder et al. (2001) and performed as described previously. Briefly, tubal endoscopic flushing was performed by accessing the oviducts using a glass capillary that was replaced by a silicon-covered flushing metal tube. Forty to sixty $\mathrm{ml} \mathrm{PBS}$ plus 1\% FCS were flushed through the oviducts and collected via an embryo flushing catheter in an embryo filter (Em Con, no. 04135; Immuno Systems Inc., Spring Valley, WI, USA). A further $300-400 \mathrm{ml}$ was used for additional flushing of the uterine horns. To get two-cell stage embryos, donor animals were flushed $48 \mathrm{~h}$ post-insemination, while eight-cell stage embryos were obtained by flushing at $72 \mathrm{~h}$ post-insemination. For the collection of blastocysts, donor cows were prepared according to the following protocol. A total of $320 \mathrm{mg}$ p-FSH (Folltropin; Bioniche Animal Health, Montréal, Quebec, Canada) was given using eight i.m. injections in decreasing concentrations over a 5-day period. Content of dominant follicles $(>6 \mathrm{~mm})$ was aspirated using an ultrasound guided system $36-48 \mathrm{~h}$ before the first FSH injection, which is any time between day 6 and day 12 of the estrous cycle. A prostaglandin injection (500 $\mu$ g estrumate; Schering, Montréal, Quebec, Canada) was given to induce the luteolysis of the corpus luteum at the eight p-FSH injections. Another prostaglandin injection was given $12 \mathrm{~h}$ after the first one. The cow was inseminated with frozen semen 12 and $24 \mathrm{~h}$ after the first signs of estrus. The embryos were recovered from the uterine horns 7 days after the first signs of estrus by flushing the uterine horns with a PBS solution. Once recovered, morphological good quality GV oocytes, two-cell embryos, eight-cell embryos, and blastocysts were washed twice in PBS, frozen in Cryotubes in groups of 10 , and stored at $-80^{\circ} \mathrm{C}$ until RNA isolation. To limit the possibility of individual effect, pools of ten specimens repeated thrice for each developmental stage for a total of 120 embryos coming from 23 heifers and four Holstein bulls were used for array analysis. Animals were cared for according to the recommended code of practice following the guidelines of the Canadian Council on Animal Care (1993).

\section{RNA extraction and amplification}

Total RNA was extracted from three pools of each developmental stage using either the Picopure RNA extraction kit (Molecular Devices Corporation, Sunnyvale, CA, USA) or the Absolutely RNA Microprep Kit (Stratagene) according to the manufacturer's instructions. Extensive validations revealed the similarity between RNA extracted from both kits (I Gilbert, S Scantland, I Dufort, M-A Sirard \& C Robert 2007, personal communication). Next, a DNAse treatment was included for the removal of genomic DNA contamination. RNA was precipitated using sodium acetate $(0.1 \mathrm{~V})$, isopropanol $(1.5 \mathrm{~V})$, and linear acrylamide $(20 \mu \mathrm{g} / \mathrm{ml})$ (Ambion, Austin, TX, USA) as a carrier. RNA was resuspended in $11 \mu$ l water and stored at $-80^{\circ} \mathrm{C}$. Total RNA was amplified by two rounds of linear amplification using the RiboAmp HS RNA Amplification kit (Molecular Devices Corporation) according to the manufacturer's instructions except the in vitro transcription lasted $80 \mathrm{~min}$ in the first round and $180 \mathrm{~min}$ in the second round to avoid the plateau phase of the reactions as determined by a time course analysis of the kinetics of the in vitro transcription reactions (Gilbert, 2009). During the second round of amplification, UTP amino-allyl (Ambion) were incorporated. The aRNA was quantified with the Nanodrop ND-1000 (NanoDrop Technologies, Wilmington, DE, USA).

\section{Probe labeling and microarray hybridization}

For every developmental stage, $2.5 \mu \mathrm{g}$ amplified amino-allyl modified RNA from each sample was used for labeling with Alexa fluor 647 (Molecular Probes, Burlington, Canada) according to the manufacturer's protocol. The labeled RNA 
was purified using the Picopure RNA extraction kit (Molecular Devices Corporation) and resuspended in $15 \mu \mathrm{l}$ elution buffer. Each probe was quantified using the Nanodrop ND-1000 (NanoDrop Technologies). A universal reference was used as a standard for our microarray experiments. This universal reference pool, composed of aRNA from GV oocytes and blastocysts obtained through the modified amplification protocol described above and DNA from a subtracted library obtained from a subtraction of bovine GV oocytes and somatic tissues (Vallee et al. 2005), was labeled with Alexa fluor 555 (Molecular Probes). Three microgram aliquots of the labeled reference were used in every hybridization.

As positive controls, GFP fragment (Vigneault et al. 2004) and $C A B 1$ gene from $A$. thaliana included in the SpotReport cDNA Array Validation System (Stratagene) were also amplified and labeled. To account for the equalization of the natural RNA content differences between developmental stages when using equal amount of aRNA for microarray probes, 100 ng GFP amino-allyl modified aRNA was added to each sample following the second round of amplification prior to labeling with Alexa fluor 647 (Molecular Probes). For the CAB1 control, 15 ng DNA labeled with Alexa fluor 555 and 647 (Molecular Probes) were added to the probes prior to hybridization.

Hybridizations were performed in triplicate on our bovine developmental microarray slide (v.1.2) containing 1153 transcripts derived from subtracted libraries (Sirard et al. 2005). Hybridizations were performed using the Slidehyb buffer \#1 (Ambion) at $50{ }^{\circ} \mathrm{C}$ for $18 \mathrm{~h}$ in the SlideBooster (Advalytix, San Francisco, CA, USA). Slides were then washed twice with $2 \times \mathrm{SSC}-0.5 \%$ SDS at $50{ }^{\circ} \mathrm{C}$ for $15 \mathrm{~min}$ and twice with $0.5 \mathrm{SSC}-0.5 \% \mathrm{SDS}$ at $50{ }^{\circ} \mathrm{C}$ for $15 \mathrm{~min}$. Next, the slides were dipped thrice in $1 \times \mathrm{SSC}$ and thrice in $\mathrm{H}_{2} \mathrm{O}$. Finally, the slides were dried by centrifugation at room temperature at $1200 \mathrm{~g}$ for $5 \mathrm{~min}$.

\section{Microarray image processing}

Slides were scanned using the VersArray ChipReader System (Bio-Rad) and visualized with the ChipReader software (Media Cybernetics, San Diego, CA, USA). Microarray image processing was performed with the ArrayPro Analyzer software (Media Cybernetics). Microarray experiments presented in this study adhere to the standards proposed by the Microarray Gene Expression Data Society. Raw and normalized data are stored in the public ArrayExpress database (www.ebi.ac.uk/ arrayexpress) under accession number E-MEXP-2075.

\section{Microarray data analysis}

A three-step strategy was used to normalize our microarray data. First, in order to remove any spatial effect on the array, an ANOVA analysis was performed on each array, by computing and subtracting any column effect (four columns of subarrays on each array), any row effect (considering only two rows of subarrays: the top 16 and the bottom 16 subarrays), and any column/row interaction. Then, in order to account for hybridization efficiency between arrays, an ANOVA analysis was performed on all the arrays using only the reference samples. An array effect was then estimated and removed from the expression of both the reference and the developmental stage expressions. Finally, in order to take into consideration the difference in RNA quantities found naturally in each developmental stage as seen in Fig. 1, the spiked-in GFP controls were used for normalization. Prior to labeling, each sample was spiked with equal amount of GFP (100 ng). Then, for each probe, an equal amount of aRNA $(2.5 \mu \mathrm{g})$ was used for hybridization. This allowed optimal hybridization, but consequently varied the quantity of embryos and GFP used for each developmental stage. The signals generated by the GFP spikes were representative of the RNA yields for each developmental stage. By normalizing our data with the signals generated by the GFP, we were able to correct the data to the natural relative RNA abundance between developmental stages. This consequently transformed the dataset on each array to account for the natural differences in RNA content that characterized the bovine early developmental stages while maintaining optimal hybridization conditions (Fig. 1). The normalized data were next uploaded in the NIA array analysis tool for analysis (Sharov et al. 2005). First background threshold was determined according to the plot of error function (s.D. (=square root of the error variance), versus expression level (log intensity)). Clones with a mean log signal intensity above the calculated background threshold (log 2.5) were considered as present. Next, a one-way ANOVA (default parameters, except FDR $<0.1$ ) was conducted. Further data processing including correlation coefficient, hierarchical clustering, and mean pair-wise comparison were also performed through NIA microarray analysis tool.

\section{DNA sequencing and clones identification}

DNA sequencing and clones identification were performed as previously described (Sirard et al. 2005). Briefly, the resulting sequence traces were visualized with the online freeware Chromas 1.45 (http://www.technelysium.com.au/chromas. html) and uploaded into a cDNA Library Manager program (coded by Genome Canada Bioinformatics) that automates and facilitates sequence analysis and clone identification (Vallee et al. 2005). For clone identification, sequence traces were uploaded into the cDNA Library Manager, trimmed (Phred software, Seattle, WA, USA) and compared against a locally installed GenBank database (Basic Local Alignment Search Tool (BLAST) http://www.ncbi.nlm.nih.gov/blast/).

\section{Quantitative PCR}

Primer sequences are shown in Table 3. For each gene examined, a representative standard curve, consisting of PCR products purified with the QIAquick PCR Purification Kit (Qiagen) and quantified with a Nanodrop ND-1000 (NanoDrop Technologies) was included in the run. The standard curve consisted of four dilutions of the purified PCR products (diluted from $0.1 \mathrm{pg}$ to $0.1 \mathrm{fg}$ ). Quantitative PCR was executed on a LightCycler apparatus using SYBR green incorporation (Roche Diagnostics). Aliquots of the same total RNA used for microarray hybridizations were reverse transcribed using 
$4 U$ of Sensiscript Reverse Transcriptase (Qiagen) in a reaction containing $1 \times$ RT buffer, $0.5 \mathrm{mM}$ dNTP, 10U RNase inhibitor (Promega) and $10 \mu \mathrm{M}$ random decamers (Ambion). The RNA was incubated with random decamers $5 \mathrm{~min}$ at $65^{\circ} \mathrm{C}$ then placed on ice. The other components were added to the reaction and incubated $10 \mathrm{~min}$ at $25{ }^{\circ} \mathrm{C}$ to allow priming of the random decamers and then $1 \mathrm{~h}$ at $37^{\circ} \mathrm{C}$. Each PCR contained cDNA corresponding to $1 / 20$ embryo and a reaction mixture consisting of $0.25 \mathrm{mM}$ of each primer, $3 \mathrm{mM} \mathrm{MgCl} 2$ in $1 \times$ SYBR green mix containing dNTPs, FastStart Taq DNA polymerase enzyme, and buffer (Roche). The PCR conditions used for all genes were as follows: denaturing cycle of $10 \mathrm{~min}$ at $95{ }^{\circ} \mathrm{C}$; 50 PCR cycles (denaturing: $95^{\circ} \mathrm{C}$ for $5 \mathrm{~s}$; annealing: specific temperature, see Table 3 , for $5 \mathrm{~s}$; extension: $72{ }^{\circ} \mathrm{C}$ for $20 \mathrm{~s})$; followed by a melting cycle. The DNA quantification was performed using LightCycler Software Version 3.5 with comparison with the standard curve. A one-way ANOVA and Tukey's test were performed using SAS Software Version 9.1 to determinate the statistically significant differences in mRNA levels between each developmental stage. The statistical significance was set at $P<0.05$. The PCR products specificity was confirmed by analysis of the melting curve given by the Lightcycler software. The products were then electrophoresed on a $1 \%$ agarose gel and were sequenced to confirm that the proper product was amplified.

\section{Declaration of interest}

The authors declare there is no conflict of interest that could be perceived as prejudicing the impartiality of the research reported.

\section{Funding}

$M V$ is supported by NSERC fellowship. This project was supported by Canada Research Chair and Natural Science and Engineering Research Council of Canada.

\section{Author contribution statement}

$\mathrm{MV}$ was involved in the conceptualization of this project, produced a subtracted library, analyzed the results, and wrote the manuscript. I D carried out the RNA extraction and amplification, labeling and hybridization of the microarray. S D performed the quantitative RT-PCR and analyzed the results. A $L$ designed and carried out the microarray data normalization. C G produced three subtracted libraries and designed and produced the bovine developmental microarray slide (v.1.2). I G designed the protocols used for the RNA amplification procedure. C R was involved in the conceptualization and realization of this project, and critically reviewed the manuscript for important intellectual content. M A S was involved in the conceptualization of this project and the preparation of the manuscript, and provided mentorship. All authors read and approved the final manuscript.

\section{Acknowledgements}

In vivo two- and eight-cell embryos were obtained through the collaboration of Dawit Tesfaye and Karl Schellander, Institute of Animal Science, Animal Breeding and Husbandry Group, University of Bonn, 53115 Bonn, Germany and $\mathrm{Dr} U$ Besenfelder, Department for Agrobiotechnology, Institute of Biotechnology in Animal Production, IFA-Tulln, BOKUUniversity of Natural Resources and Applied Life Sciences, Veterinaerplatz 1, A-1210 Vienna, Austria.

\section{References}

Adjaye J, Herwig R, Herrmann D, Wruck W, Benkahla A, Brink TC, Nowak M, Carnwath JW, Hultschig C, Niemann H et al. 2004 Crossspecies hybridisation of human and bovine orthologous genes on high density cDNA microarrays. BMC Genomics 583.

Adjaye J, Herwig R, Brink TC, Herrmann D, Greber B, Sudheer S, Groth D, Carnwath JW, Lehrach H \& Niemann H 2007 Conserved molecular portraits of bovine and human blastocysts as a consequence of the transition from maternal to embryonic control of gene expression. Physiological Genomics 31 315-327.

Aiba K, Sharov AA, Carter MG, Foroni C, Vescovi AL \& Ko MS 2005 Defining a developmental path to neural fate by global expression profiling of mouse embryonic stem cells and adult neural stem/ progenitor cells. Stem Cells 4 889-895.

Bachvarova R, De Leon V, Johnson A, Kaplan G \& Paynton BV 1985 Changes in total RNA, polyadenylated RNA, and actin mRNA during meiotic maturation of mouse oocytes. Developmental Biology 108 325-331.

Besenfelder U, Havlicek V, Mosslacher G \& Brem G 2001 Collection of tubal stage bovine embryos by means of endoscopy. A technique report. Theriogenology 55 837-845.

Canadian Council on Animal Care 1993 Guide to the Care and Use of Experimental Animals, Ottawa: Canadian Council on Animal Care.

Carter MG, Hamatani T, Sharov AA, Carmack CE, Qian Y, Aiba K, Ko NT, Dudekula DB, Brzoska PM, Hwang SS et al. 2003 In situ-synthesized novel microarray optimized for mouse stem cell and early developmental expression profiling. Genome Research 13 1011-1021.

Dalbies-Tran R \& Mermillod P 2003 Use of heterologous complementary DNA array screening to analyze bovine oocyte transcriptome and its evolution during in vitro maturation. Biology of Reproduction $\mathbf{6 8}$ 252-261.

Dalbies-Tran R, Papillier P, Pennetier S, Uzbekova S \& Monget P 2005 Bovine mater-like NALP9 is an oocyte marker gene. Molecular Reproduction and Development 71 414-421.

Dessie SW, Rings F, Holker M, Gilles M, Jennen D, Tholen E, Havlicek V, Besenfelder U, Sukhorukov VL, Zimmermann U et al. 2007 Dielectrophoretic behavior of in vitro-derived bovine metaphase II oocytes and zygotes and its relation to in vitro embryonic developmental competence and mRNA expression pattern. Reproduction 133 931-946.

Epifano O, Liang LF \& Dean J 1995 Mouse Zp1 encodes a zona pellucida protein homologous to egg envelope proteins in mammals and fish. Journal of Biological Chemistry 270 27254-27258.

Fair T, Carter F, Park S, Evans AC \& Lonergan P 2007 Global gene expression analysis during bovine oocyte in vitro maturation. Theriogenology 68 S91-S97.

Ghanem N, Holker M, Rings F, Jennen D, Tholen E, Sirard MA, Torner H, Kanitz W, Schellander K \& Tesfaye D 2007 Alterations in transcript abundance of bovine oocytes recovered at growth and dominance phases of the first follicular wave. BMC Developmental Biology 790.

Gilbert I, Scantland S, Dufort I, Gordynska O, Labbe A, Sirard MA \& Robert C 2009 Real-time monitoring of aRNA production during T7 amplification to prevent the loss of sample representation during microarray probe preparation. Nucleic Acids Research 37 e65.

Hamatani T, Carter MG, Sharov AA \& Ko MS 2004a Dynamics of global gene expression changes during mouse preimplantation development. Developmental Cell 6 117-131. 
Hamatani T, Daikoku T, Wang H, Matsumoto H, Carter MG, Ko MS \& Dey SK 2004b Global gene expression analysis identifies molecular pathways distinguishing blastocyst dormancy and activation. PNAS $\mathbf{1 0 1}$ 10326-10331.

Hamatani T, Falco G, Carter MG, Akutsu H, Stagg CA, Sharov AA, Dudekula DB, VanBuren V \& Ko MS 2004c Age-associated alteration of gene expression patterns in mouse oocytes. Human Molecular Genetics 13 2263-2278.

Khatri P, Draghici S, Ostermeier GC \& Krawetz SA 2002 Profiling gene expression using onto-express. Genomics 79 266-270.

Ko MS, Kitchen JR, Wang X, Threat TA, Hasegawa A, Sun T, Grahovac MJ, Kargul GJ, Lim MK, Cui Y et al. 2000 Large-scale cDNA analysis reveals phased gene expression patterns during preimplantation mouse development. Development 127 1737-1749.

Liang LF, Chamow SM \& Dean J 1990 Oocyte-specific expression of mouse Zp-2: developmental regulation of the zona pellucida genes. Molecular and Cellular Biology 10 1507-1515.

Liang L, Soyal SM \& Dean J 1997 FIGalpha, a germ cell specific transcription factor involved in the coordinate expression of the zona pellucida genes. Development 124 4939-4947.

Memili E, Dominko T \& First NL 1998 Onset of transcription in bovine oocytes and preimplantation embryos. Molecular Reproduction and Development 51 36-41.

Pennetier S, Uzbekova S, Guyader-Joly C, Humblot P, Mermillod P \& Dalbies-Tran R 2005 Genes preferentially expressed in bovine oocytes revealed by subtractive and suppressive hybridization. Biology of Reproduction 73 713-720.

Philpott CC, Ringuette MJ \& Dean J 1987 Oocyte-specific expression and developmental regulation of ZP3, the sperm receptor of the mouse zona pellucida. Developmental Biology $121568-575$.

Piko L \& Clegg KB 1982 Quantitative changes in total RNA, total poly(A), and ribosomes in early mouse embryos. Developmental Biology $\mathbf{8 9}$ 362-378.

Robert C, Barnes FL, Hue I \& Sirard MA 2000 Subtractive hybridization used to identify mRNA associated with the maturation of bovine oocytes. Molecular Reproduction and Development 57 167-175.

Sharov AA, Dudekula DB \& Ko MS 2005 A web-based tool for principal component and significance analysis of microarray data. Bioinformatics 21 2548-2549.

Sirard MA, Dufort I, Vallee M, Massicotte L, Gravel C, Reghenas H, Watson AJ, King WA \& Robert C 2005 Potential and limitations of bovine-specific arrays for the analysis of mRNA levels in early development: preliminary analysis using a bovine embryonic array. Reproduction, Fertility, and Development 17 47-57.

Su YQ, Sugiura K, Woo Y, Wigglesworth K, Kamdar S, Affourtit J \& Eppig JJ 2007 Selective degradation of transcripts during meiotic maturation of mouse oocytes. Developmental Biology 302 104-117.
Telford NA, Watson AJ \& Schultz GA 1990 Transition from maternal to embryonic control in early mammalian development: a comparison of several species. Molecular Reproduction and Development 26 90-100.

Vallee M, Gravel C, Palin MF, Reghenas H, Stothard P, Wishart DS \& Sirard MA 2005 Identification of novel and known oocyte-specific genes using complementary DNA subtraction and microarray analysis in three different species. Biology of Reproduction 73 63-71.

Vallee M, Robert C, Methot S, Palin MF \& Sirard MA 2006 Cross-species hybridizations on a multi-species CDNA microarray to identify evolutionarily conserved genes expressed in oocytes. BMC Genomics 7113.

Vallee M, Aiba K, Piao Y, Palin M-F, Ko MS \& Sirard M-A 2008 Comparative analysis of oocyte transcript profiles reveals a high degree of conservation among species. Reproduction 135 439-448.

Vigneault C, McGraw S, Massicotte L \& Sirard MA 2004 Transcription factor expression patterns in bovine in vitro-derived embryos prior to maternal-zygotic transition. Biology of Reproduction 70 1701-1709.

Wang QT, Piotrowska K, Ciemerych MA, Milenkovic L, Scott MP, Davis RW \& Zernicka-Goetz M 2004 A genome-wide study of gene activity reveals developmental signaling pathways in the preimplantation mouse embryo. Developmental Cell 6 133-144.

Wassarman PM \& Kinloch RA 1992 Gene expression during oogenesis in mice. Mutation Research 296 3-15.

Whitworth KM, Agca C, Kim JG, Patel RV, Springer GK, Bivens NJ, Forrester LJ, Mathialagan N, Green JA \& Prather RS 2005 Transcriptional profiling of pig embryogenesis by using a 15-K member unigene set specific for pig reproductive tissues and embryos. Biology of Reproduction 72 1437-1451.

Yao J, Ren X, Ireland JJ, Coussens PM, Smith TP \& Smith GW 2004 Generation of a bovine oocyte cDNA library and microarray: resources for identification of genes important for follicular development and early embryogenesis. Physiological Genomics 19 84-92.

Yoon SJ, Kim KH, Chung HM, Choi DH, Lee WS, Cha KY \& Lee KA 2006 Gene expression profiling of early follicular development in primordial, primary, and secondary follicles. Fertility and Sterility 85 193-203.

Zeng F \& Schultz RM 2005 RNA transcript profiling during zygotic gene activation in the preimplantation mouse embryo. Developmental Biology 283 40-57.

Zeng F, Baldwin DA \& Schultz RM 2004 Transcript profiling during preimplantation mouse development. Developmental Biology 272 483-496.

Received 23 December 2008

First decision 18 February 2009

Revised manuscript received 5 April 2009

Accepted 21 April 2009 